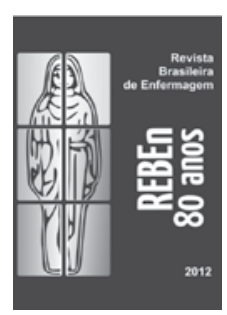

\title{
Atributos essenciais da Atenção Primária e a Estratégia Saúde da Família
}

\author{
Primary Health Care essential attributes and the Family Health Strategy
}

Atributos esenciales de la Atención Primaria y la Estrategia Salud de la Familia

\begin{abstract}
Maria Amélia de Campos Oliveira', Iara Cristina Pereira"
' Universidade de São Paulo, Escola de Enfermagem, Departamento de Enfermagem em Saúde Coletiva. São Paulo-SP, Brasil.

"Universidade Federal de Mato Grosso do Sul, Escola de Enfermagem. Campo Grande-MS, Brasil. Universidade de São Paulo, Escola de Enfermagem, Programa de Pós-Graduação em Enfermagem (Doutoranda). São Paulo-SP, Brasil.
\end{abstract}

Submissão: 02-09-2013 Aprovação: 04-09-2013

\section{RESUMO}

A Atenção Primária à Saúde tem como atributos essenciais a atenção no primeiro contato, a longitudinalidade, a integralidade e a coordenação, e como atributos derivados a orientação familiar e comunitária e a competência cultural. Discute-se a importância de tais atributos na Estratégia Saúde da Família, proposta político-governamental para a mudança do modelo de atenção à saúde no contexto do Sistema Único de Saúde no Brasil.

Descritores: Política de Saúde; Atenção Primária de Saúde; Saúde da Família.

ABSTRACT
The essential attributes of Primary Health Care are attention at first contact, longitudinality, completeness and coordination, and the derived attributes are family and community orientation and cultural competence. This paper discusses the presence of such attributes in Family Health Strategy, a political and governmental proposal to change the health care model in the context of the Unified Health System in Brazil.

Descriptors: Health Policy; Primary Health Care; Family Health.

\section{RESUMEN}

La Atención Primaria de Salud tiene como atributos esenciales la atención en el primer contacto, la longitudinalidad, la integridad y la coordinación, y como atributos derivados, la orientación familiar y comunitaria y la competencia cultural. Se discute la presencia de estos atributos en la Estrategia Salud de la Familia, propuesta política de gobierno para cambiar el modelo de atención de la salud en el contexto del Sistema Único de Salud en Brasil.

Descriptores: Política de Salud; Atención Primaria de Salud; Salud de la Familia. 


\section{INTRODUÇÃO}

A Atenção Primária em Saúde (APS) é reconhecidamente um componente-chave dos sistemas de saúde. Esse reconhecimento fundamenta-se nas evidências de seu impacto na saúde e no desenvolvimento da população nos países que a adotaram como base para seus sistemas de saúde: melhores indicadores de saúde, maior eficiência no fluxo dos usuários dentro do sistema, tratamento mais efetivo de condições crônicas, maior eficiência do cuidado, maior utilização de práticas preventivas, maior satisfação dos usuários e diminuição das iniquidades sobre o acesso aos serviços e o estado geral de saúde ${ }^{(1-2)}$.

A APS apresenta dois aspectos distintos e interdependentes: é uma estratégia de organização e reorganização dos sistemas de saúde, nos quais representa o primeiro nível de atenção, e também um modelo de mudança da prática clínico-assistencial dos profissionais de saúde. Orienta-se por eixos estruturantes que, na literatura internacional, recebem o nome de atributos essenciais: atenção ao primeiro contato, longitudinalidade, integralidade e coordenação; e atributos derivados: orientação familiar e comunitária e competência cultural ${ }^{(1)}$.

No caso brasileiro, a opção pelo combate às iniquidades em saúde elevou a APS à condição de reordenadora do sistema de atenção à saúde. No Brasil, foi adotada a designação Atenção Básica, para contrapor-se à perspectiva assumida por muitos países e organismos internacionais, como o Banco Mundial, que entendem a atenção primária como um conjunto de ações de saúde de baixa complexidade, dedicada a populações de baixa renda, no sentindo de minimizar a exclusão social e econômica decorrentes da expansão do capitalismo global ${ }^{(3)}$.

A designação Atenção Básica, tomada pelo governo brasileiro, objetiva, portanto contrapor-se a proposta político-ideológica da atenção primária seletiva destinada às populações pobres, e busca resgatar o caráter universalista da Declaração de Alma-Ata enfatizando o papel de reorientação do modelo assistencial para um sistema universal e integrado de atenção à saúde, que engloba diferentes setores, públicos e privados, com e sem fins lucrativos, o Sistema Único de Saúde (SUS)*

Juridicamente, a Constituição de 1988 foi o marco conceitual que consolidou a nova consciência social de promoção de saúde que deu origem ao SUS, a maior conquista do movimento sanitário brasileiro. Com o SUS, estabeleceu-se uma nova relação entre o Estado e a sociedade civil em prol do acesso pleno aos serviços em busca de um novo padrão de cidadania(4).

A consolidação do SUS apoia-se no entendimento de que os serviços de saúde devem estar organizados a partir de uma rede de cuidados articulada, com fluxos conhecidos e regulados, cujo objetivo é acolher necessidades sentidas por usuários, gestores e sociedade, definidas por critérios epidemiológicos, econômicos e culturais(4)

Entretanto, os serviços públicos de saúde no Brasil ainda se caracterizam pela fragmentação e descontinuidade assistencial, em decorrência de fragilidades na articulação entre as instâncias gestoras do sistema e a gerência dos serviços; entre os serviços de saúde e de apoio diagnóstico e terapêutico e entre as práticas clínicas desenvolvidas por diferentes profissionais de um ou mais serviços, voltadas a um mesmo indivíduo ou grupo de indivíduos ${ }^{(5)}$.

A fragmentação da atenção e a responsabilização clínica insuficiente são reflexos do modo de organizar o trabalho e os processos de decisão nos serviços de saúde. Essa (ir)racionalidade gerencial induz tais serviços a se responsabilizar pelos usuários apenas enquanto estão dentro de seu espaço físico - nem antes de entrarem nem depois de saírem, e pouco contribui para a responsabilização sanitária territorial das populações ${ }^{(5)}$.

O financiamento das ações realizadas pelos serviços de saúde ainda está voltado principalmente para a dimensão quantitativa (número de consultas e procedimentos por período de trabalho) e são raros os contratos com base em encargos sobre uma população que habite certo território e que valorizem atividades em conjunto entre os diversos serviços da rede para pacientes compartilhados ${ }^{(5)}$.

Diante da tendência de fragmentar a clínica tanto internamente a cada serviço, quanto externamente, nos diversos níveis do sistema de saúde, vários esforços têm sido realizados pelo governo federal para estimular a qualidade dos serviços de saúde e garantir o acesso dos usuários. A Estratégia Saúde da Família (ESF) é uma das propostas do Ministério da Saúde para a reorganização da Atenção Primária, podendo ser considerada uma alternativa de ação para o alcance dos objetivos de universalização, equidade e integralidade.

Este estudo propõe-se a realizar uma reflexão teórica sobre a presença dos atributos essenciais da APS na ESF.

\section{Os atributos da APS e a Estratégia Saúde da Família}

A organização dos serviços de saúde da Atenção Primária por meio da ESF prioriza ações de promoção, proteção e recuperação de saúde, de forma integral e continuada. Em expansão por todo o território nacional, a ESF define-se por um conjunto de ações e serviços que vão além da assistência médica, estruturando-se com base no reconhecimento das necessidades da população, apreendidas a partir do estabelecimento de vínculos entre os usuários dos serviços e os profissionais de saúde, em contato permanente com o território.

A ESF propõe que a atenção à saúde centre-se na família, entendida e percebida a partir de seu ambiente físico e social, o que leva os profissionais de saúde a entrar em contato com as condições de vida e saúde das populações, permitindo-lhes uma compreensão ampliada do processo saúde-doença e da necessidade de intervenções que vão além das práticas curativas. Para tanto, os profissionais que nela atuam deverão dispor de um arsenal de recursos tecnológicos bastante diversificados e complexos.

Ao considerar a família como objeto de atenção, a ESF está contemplando dois atributos derivados da APS: a orientação

* Esclarecemos que, no decorrer de grande parte deste artigo, estaremos utilizando a expressão Atenção Primária ou Atenção Primária em Saúde, porque são nomes estabelecidos mundialmente, referência fundamental para as reformas sanitárias particularmente após a realização da I Conferência Internacional sobre Cuidados Primários de Saúde, em Alma-Ata no ano de 1978. 
familiar/comunitária e a competência cultural, que pressupõem o reconhecimento das necessidades familiares em função do contexto físico, econômico e cultural.

\section{Atenção no primeiro contato}

A expressão primeiro contato implica acessibilidade e utilização dos serviços de saúde pelos usuários a cada novo problema ou a cada novo episódio de um mesmo problema ${ }^{(1)}$. Em outras palavras, o primeiro contato pode ser definido como porta de entrada dos serviços de saúde, ou seja, quando a população e a equipe identificam aquele serviço como o primeiro recurso a ser buscado quando há uma necessidade ou problema de saúde.

Embora acesso e acessibilidade frequentemente sejam utilizados como sinônimos têm significados diferentes, ainda que complementares. A acessibilidade refere-se às características da oferta que possibilitam que as pessoas cheguem aos serviços, enquanto o acesso é a forma como as pessoas percebem a acessibilidade. $O$ conceito de acesso traz consigo a ideia de não o restringir a entrada nos serviços de saúde, enquanto a acessibilidade diz respeito à oferta de serviços, à capacidade de produzir serviços e responder às necessidades de saúde de uma determinada população( ${ }^{(6)}$. Acessibilidade pode ser conceituada então como a capacidade do usuário obter cuidados de saúde sempre que necessitar e de maneira fácil e conveniente.

A acessibilidade deve ser considerada com relação aos aspectos geográficos, organizacionais, socioculturais e econômicos. O estudo da acessibilidade é primordial para permitir a identificação dos aspectos que podem se tornar obstáculos, assim como dos que facilitam a busca pelo atendimento à saúde ${ }^{(6)}$.

A acessibilidade geográfica reflete a distância entre a população e os recursos, podendo ser medida por distância, tempo de deslocamento, custo do transporte, entre outros condicionantes. A acessibilidade organizacional abrange as características ligadas ao modo de organização dos serviços de saúde que obstaculizam ou facilitam a capacidade das pessoas na utilização dos mesmos. Inclui aspectos tais como: tempo para obter uma consulta, tipo de agendamento, turnos de funcionamento, tempo para fazer exames laboratoriais, continuidade do tratamento. Os aspectos socioculturais e econômicos da acessibilidade incluem o conhecimento de sinais e sintomas, a percepção do risco de gravidade, o conhecimento sobre o próprio corpo e sobre oferta dos serviços de saúde, medo do diagnóstico, crenças, hábitos, dificuldades de comunicação com a equipe de saúde, crédito dado ao sistema de saúde, graus de instrução, emprego, renda e seguridade social|(6).

Oficialmente, a Atenção Primária configura-se como a porta de entrada do sistema de saúde brasileiro. Espera-se que os serviços desse nível de atenção sejam acessíveis e resolutivos frente às principais necessidades de saúde trazidas pela população. A adoção de ferramentas apropriadas de trabalho gerencial, tais como a abordagem multidisciplinar, o planejamento das ações, a organização horizontal do trabalho e o compartilhamento do processo decisório podem contribuir significativamente para oferecer atenção ao primeiro contato.

\section{Longitudinalidade}

A longitudinalidade implica a existência de uma fonte regular de atenção e seu uso ao longo do tempo, independente da presença de problemas específicos relacionados à saúde ou do tipo de problema ${ }^{(1)}$. Na literatura internacional, o termo continuidade do cuidado é utilizado com sentido semelhante à palavra longitudinalidade, embora esses termos possuam especificidades conceituais. Ou seja: mesmo que ocorram interrupções na continuidade da atenção, isto não significa que a relação pessoal de longa duração entre os profissionais de saúde e os usuários não exista ou que seja interrompida ${ }^{(7-8)}$.

Uma relação pessoal de longa duração entre os profissionais de saúde e os pacientes em suas unidades de saúde é denominada longitudinalidade temporal, porém sua avaliação é controversa, pois uma relação de longa duração pode se estabelecer mediante relacionamento interpessoal insatisfatório, enquanto um bom relacionamento pode ocorrer em curto período de tempo ${ }^{(8)} \mathrm{Em}$ decorrência disso, os estudos sobre longitudinalidade têm se voltado para investigar o vínculo interpessoal entre usuários e sua fonte de atenção (longitudinalidade pessoal) cujas características compreendem: o alcance do entendimento entre profissionais e usuários; a tranqüilidade destes em falar aos profissionais a respeito de suas preocupações e o quanto sentem que o profissional está interessado em outros aspectos de sua vida e não apenas em seu problema de saúde e que entende quais problemas são mais importantes para eles ${ }^{(1)}$.

A presença do atributo de longitudinalidade tende a produzir diagnósticos e tratamentos mais precisos, que reduzem os encaminhamentos desnecessários para especialistas e a realização de procedimentos de maior complexidade.

A formação de profissionais de saúde, quando desvinculada da realidade das condições de vida e saúde da população, resulta em falta de preparo para lidar com pacientes com distintas características socioculturais, o que constitui um obstáculo ao alcance da longitudinalidade pessoal na ESF.

Para prover a sociedade brasileira de profissionais habilitados para responder às necessidades de saúde da população, o Ministério da Saúde deu início em 2004 ao Programa Nacional de Humanização (PNH), que, entre suas diretrizes e dispositivos, define uma estratégia de modificação do processo de trabalho em saúde. Uma das ferramentas propostas para esta modificação é o acolhimento.

A proposta do acolhimento constitui um elemento da mudança no processo de trabalho em saúde, com potencial de ampliar as práticas de cuidado ${ }^{(9-10)}$. O acolhimento não é necessariamente uma atividade em si, mas uma atitude que permeia toda atividade assistencial. Consiste na busca constante de reconhecimento das necessidades de saúde dos usuários e das formas possíveis de satisfazê-las, o que resulta em encaminhamentos, deslocamentos e trânsitos pela rede assistencial ${ }^{(10)}$.

A tecnologia do acolhimento pode ser considerada uma reforma nos processos de trabalho e no relacionamento entre profissionais e usuários e, portanto, uma estratégia para o alcance da longitudinalidade pessoal. Está fortemente relacionada à boa comunicação e tende a favorecer a continuidade e a efetividade do cuidado, contribuindo para a implementação 
de ações de promoção e de prevenção de agravos. Para sua efetivação faz-se necessário o comprometimento dos trabalhadores, de modo que sejam desenvolvidas práticas que visem ao atendimento das necessidades de saúde da população, por meio de atos de fala, escuta, vínculo e negociação.

\section{Integralidade}

A integralidade é um dos pilares na construção do SUS consagrado pela Constituição Federal de 1988 e possui quatro dimensões: primazia das ações de promoção e prevenção, atenção nos três níveis de complexidade da assistência médica, articulação das ações de promoção, proteção e prevenção e abordagem integral do indivíduo e das famílias ${ }^{(11)}$.

A primeira reafirma a existência de um movimento crítico e de recusa a um sistema de saúde que privilegia a especialização e a medicalização. A promoção de saúde pressupõe uma concepção que não restrinja a saúde à ausência de doença, mas que seja capaz de atuar sobre seus determinantes que são as condições sociais em que as pessoas vivem e trabalham.

Esta concepção implica potencializar formas mais amplas de intervir em saúde, exigindo e desafiando a construção de ações intersetoriais, pois o processo saúde-adoecimento é decorrência de múltiplos aspectos, sendo pertinente a todos os setores da sociedade. Implica paralelamente, por parte do Estado, a formulação de políticas sociais e econômicas que extrapolem o enfoque de risco e atuem na diminuição das desigualdades sociais e melhoria das condições de vida.

A segunda dimensão toma a integralidade como garantia de acesso a diversos níveis de atenção, cuja característica básica é a diferença nas densidades tecnológicas da assistência à saúde. Pressupõe, portanto a existência de uma rede de serviços em distintos níveis de complexidade e de competências, em que a integração entre as ações nos diversos níveis deve satisfazer o conjunto de cuidados demandados por um indivíduo.

Publicações do Ministério da Saúde, a exemplo do documento do MS/SESUS/1990(12), descrevem a integralidade como um princípio que pode ser contemplado por meio de um modelo assistencial organizado de forma hierarquizada e descentralizada, com sistemas formais de referência e contrarreferência, cuja garantia da integração dos recursos de infraestrutura é fundamental. A atenção integral depende de uma rede articulada de tal forma que os problemas apresentados pelos indivíduos possam ser abordados em todos os níveis de assistência necessários para a sua resolução e que o acesso a estes diferentes níveis seja harmonioso e ágil.

A terceira dimensão da integralidade é a articulação das ações de promoção, prevenção e recuperação ${ }^{(11)}$. Estas práticas de saúde tradicionalmente têm sido desenvolvidas separadamente, como ações de saúde coletiva ou como ações de atenção clínica e individual, sendo necessária uma nova forma de organizar e agir em saúde com a constituição de saberes e de ações que se interpenetrem.

O último sentido ou dimensão da integralidade é a abordagem integral do indivíduo e da família, relacionada a um valor a ser sustentado e defendido nas práticas dos profissionais de saúde, ou seja, um valor que se expressa na forma como os profissionais respondem aos que os procuram.
A integralidade do cuidado depende da redefinição de práticas, de modo a criar vínculo, acolhimento e autonomia, o que valoriza as subjetividades inerentes ao trabalho em saúde e às necessidades singulares dos sujeitos, como pontos de partida para qualquer intervenção, construindo a possibilidade do cuidado centrado no usuário. Exercer a integralidade passa pela necessidade de repensar práticas e conformações dos serviços públicos de saúde, ainda hoje caracterizados pela descontinuidade assistencial.

\section{Coordenação}

A coordenação entre níveis assistenciais pode ser definida como a articulação entre os diversos serviços e ações de saúde, de forma que estejam sincronizados e voltados ao alcance de um objetivo comum, independentemente do local onde sejam prestados. Objetiva ofertar ao usuário um conjunto de serviços e informações que respondam a suas necessidades de saúde de forma integrada, por meio de diferentes pontos da rede de atenção à saúde.

Os quatro atributos são fundamentais para que a APS assuma a coordenação das redes de atenção à saúde ${ }^{(13)}$. Contudo, a coordenação tem maior importância relativa, já que, sem ela, o primeiro contato tornar-se-ia uma função puramente administrativa, a longitudinalidade perderia muito de seu potencial e a integralidade ficaria comprometida.

A atenção coordenada é justificada e necessária, porque é cada vez maior o número de pessoas que sofrem de mais de uma doença e recebem cuidados de saúde por trabalhadores de diferentes especialidades. Necessidades de saúde são bastante complexas e em geral não são adequadamente tratadas por sistemas de saúde caracterizados pela especialização e orientação profissional isolada. A inadequação pode resultar não só em necessidades não atendidas, como também em tratamentos desnecessários, duplicidade de ações e medicalização excessiva.

Oferecer aos usuários que necessitam de cuidados mais complexos as orientações necessárias para encontrar seu caminho através do sistema de saúde só poderão ocorrer se existir coordenação do cuidado, ou seja, a capacidade de garantir a continuidade da atenção, por meio de equipes de saúde, com o reconhecimento dos problemas que requerem seguimento constante ${ }^{(13)}$.

Nesse sentido, foi instituída pelo Ministério da Saúde a Portaria n. 4.279, de 30 de Dezembro de $2010^{(14)}$, que estabelece diretrizes para a organização da Rede de Atenção à Saúde no âmbito do Sistema Único de Saúde e cujo objetivo geral é assegurar ao usuário ações e serviços de forma efetiva, contínua, integral e humanizada.

A operacionalização das redes de atenção em saúde dá-se pela interação de três elementos constitutivos: população adstrita a uma determinada região de saúde, estrutura operacional, que inclui pontos de atenção e ligações entre os pontos de atenção e um modelo de atenção à saúde.

Os pontos de atenção em saúde compreendem as unidades de APS (ou equipe da ESF), com a função de atuar como centros de comunicação, os serviços de atenção secundária e terciária e os de apoio diagnóstico e terapêutico. A conexão entre os diversos pontos de atenção é feita por 
sistemas logísticos e de governança, na forma de comissões intergestoras.

A integração das unidades de saúde da família à rede assistencial é fundamental para garantir uma oferta abrangente de serviços e para coordenar as diversas ações requeridas para resolver as necessidades menos freqüentes e mais complexas.

\section{DISCUSSÃO}

O Brasil vive uma situação de transição demográfica acelerada, acompanhada de uma transição epidemiológica singular, caracterizada pela tripla carga de doenças: persistência de doenças parasitárias, infecciosas e desnutrição, problemas de saúde reprodutiva, com mortes maternas e óbitos infantis por causas consideradas evitáveis e o desafio das doenças crônicas, que requerem cuidados de longa duração e uma abordagem sistemática para o tratamento. Os serviços de saúde brasileiros estão sendo desafiados a dar respostas contínuas e integradas e um sistema de saúde fragmentado e voltado para as condições agudas já não responde às necessidades do País.

Um dos grandes desafios do Sistema Único de Saúde é qualificar a APS para exercer a coordenação do cuidado e organizar pontos de atenção especializada integrados, intercomunicantes, capazes de assegurar que a linha de cuidado integral seja plenamente articulada com a APS e forneça aos usuários do SUS respostas adequadas as suas necessidades.

Uma iniciativa governamental foi a formulação da Política Nacional de Promoção da Saúde (PNPS), cuja institucionalização deu-se por meio da Portaria 687 GM/MS, de 30 de março de $2006^{(15)}$. O objetivo geral desta política é promover a qualidade de vida da população por meio de ações integradas e intersetoriais, de tal modo que os setores privados e os governamentais, bem como os não governamentais, juntamente com a sociedade civil, participem em conjunto do debate sobre os determinantes sociais de saúde e potencializem formas ampliadas de intervir em saúde.

Com isso, os órgãos gestores do SUS reconheceriam a importância da promoção da saúde para a equidade, e passariam a atuar para fortalecer a participação social, adotando práticas horizontais de gestão, estabelecendo redes de cooperação intersetorial, uma tarefa desafiadora e complexa, tanto do ponto de vista político quanto técnico, porque muitas ações envolvem instâncias que se encontram fora do setor saúde, implicando o estabelecimento de agendas públicas compartilhadas entre diversos setores. Seriam ainda necessárias as iniciativas de promoção da saúde junto a trabalhadores e usuários do SUS, mediante metodologias participativas que promovessem a aproximação entre os conhecimentos científicos e os saber populares e tradicionais.

A Portaria $n^{\circ} 1.559$, de $1^{\circ}$ de agosto de 2008, que instituiu a Política Nacional de Regulação do Ministério da Saúde ${ }^{(16)}$, também é uma evidência do caráter integrador da ação ministerial. Foi estruturada em três eixos articulados: a garantia da alocação de recursos para a implantação dos complexos reguladores do acesso à assistência, o desenvolvimento de instrumentos para viabilizar as funções reguladoras e o desenvolvimento de um programa de capacitação permanente de recursos humanos. Ao garantir o acesso dos usuários aos serviços de saúde, a regulação atua sobre a oferta, assume o planejamento das ações e estabelece a adequação da oferta de acordo com as necessidades identificadas.

Atualmente, o Ministério da Saúde vem se dedicando à avaliação da APS no Brasil. A Coordenação de Acompanhamento e Avaliação da Atenção Básica (CAA/DAB) tem publicado vários documentos cujo propósito é fornecer suporte aos processos decisórios no âmbito do Sistema de Saúde. Além destas publicações, têm sido desenvolvidos vários estudos sobre avaliação em saúde por pesquisadores de instituições governamentais e não governamentais.

Investigação conduzida nos municípios de Aracaju, Belo Horizonte, Florianópolis e Vitória sobre a implantação da ESF com foco na integração à rede assistencial, identificou condições facilitadores e limitantes com relação à integração dos serviços. Dentre os facilitadores são citados a implantação do sistema informatizado de marcação e de regulação de consultas e exames especializados (SISREG), a existência de prontuários eletrônicos com acesso on line, a qualificação dos gerentes e profissionais da ESF e as estratégias de educação permanente ${ }^{(17)}$.

Os fatores limitantes estão relacionados à insuficiente oferta de atenção especializada, agravada pela baixa integração com prestadores estaduais e a falta de interação pessoal entre generalistas e especialistas, o que corrobora para o maior o isolamento entre atenção básica e especializada. Ainda que a coordenação dos cuidados no âmbito gerencial nem sempre tenha revertido em ações concretas, o estudo concluiu que pode ser melhorada por meio do desenvolvimento de protocolos assistenciais e construção das linhas de cuidado ${ }^{(17)}$.

Fragilidades para a efetivação da APS no Brasil também foram constatadas por dois pesquisadores espanhóis que avaliaram a ESF em 2011, tendo visitado 70 centros de saúde em 32 cidades de 19 estados brasileiros. Dentre as falhas identificadas estão: a falta de desenvolvimento tecnológico, gerencial e científico da ESF, a visão vertical de programas e protocolos que compartimentam a prática clínica, perpetuando um modelo rígido e fragmentado de atendimento, a ênfase na "visão preventiva" que acarreta o escasso desenvolvimento da atividade clínica curativa, o encaminhamento excessivo aos especialistas e urgências, a utilização rotineira exagerada dos recursos disponíveis e a formas muito variáveis de contratação de pessoal, salários e incentivos, que não promove a fixação dos profissionais, que poderia ser obtida por meio de uma política de fomento do trabalho com dedicação exclusiva ${ }^{(18)}$.

Identificaram várias carências, entre as quais destacaram a falta de prestação de uma ampla categoria de serviços curativos e preventivos, rotineiros e de urgência nas unidades da ESF ou no domicílio, o que a implantação das unidades de Pronto Atendimento e o sucesso dos diferentes planos de saúde privados. Além disso, ressaltaram a escassa coordenação entre níveis de atendimento, gerando duplicidade de serviços, a falta de médicos da família qualificados, as equipes incompletas, principalmente por ausência do médico, a falta de equipes funcionais às quais possam ser delegadas funções e responsabilidades, de forma que cada profissional enfrente casos complexos apropriados a sua formação ${ }^{(18)}$. 
Recentemente, o Ministério da Saúde lançou o Programa Nacional de Melhoria do Acesso e da Qualidade da Atenção Básica -PMAQ-AB ${ }^{(19)}$, cujo objetivo é induzir a ampliação do acesso e a melhoria da qualidade da Atenção Básica. Algumas diretrizes da Portaria $\mathrm{n}^{\circ} 1.654$, publicada no Diário Oficial da União em 19 de julho de 2011, e que compõem a PMAQ-AB são: estimular processos contínuos e progressivos de melhoria de padrões e indicadores de acesso e qualidade, construir parâmetros de comparação entre as equipes de saúde da Atenção Básica, mobilizar, responsabilizar e envolver gestores federais, estaduais e municipais, as equipes de saúde e os usuários num processo de mudança e qualificação da Atenção Básica. Esta avaliação ainda está em curso no País.

\section{CONSIDERAÇÕES FINAIS}

Embora a APS seja considerada imprescindível para a efetividade dos sistemas de saúde, ainda enfrenta muitos desafios para que possa desempenhar seu papel de organizadora do sistema e coordenadora do cuidado em saúde. A carência de infraestrutura adequada nas unidades da ESF, a baixa densidade tecnológica, a falta de profissionalização da gestão, a ausência de equipes multiprofissionais, a precarização nas relações de trabalho, a fragilidade do modelo de atenção para dar conta de uma situação com forte prevalência de condições crônicas, a fragmentação da oferta de ações e serviços de saúde e o subfinanciamento são os principais entraves para que a APS seja uma realidade no Brasil (20).

Esta realidade é em grande parte decorrência de um contexto adverso a partir do início da década de 90, caracterizado por reformas e ajustes estruturais impostos pelas políticas de estabilização econômica. Os efeitos da globalização nas políticas de saúde são evidenciados pelo baixo patamar de financiamento público, persistência de segmentação no sistema, fraca integração dos serviços da Atenção Primária com outros níveis de atenção e acentuado processo de precarização do trabalho no setor saúde, particularmente nas formas de contratação das equipes da ESF.

Entretanto, a ESF foi assumida pelo governo brasileiro como uma proposta de reorganização do sistema de saúde na perspectiva da implementação de uma APS abrangente. E, se a opção política é por uma APS não simplificada, que forme a base e determine o trabalho de todos os outros níveis do sistema de saúde, torna-se necessário que seja assumida de forma integral e resolutiva, apesar das crises políticas e econômicas que constituem verdadeiros obstáculos à resposta do Estado e das instituições aos problemas do acesso, da prestação e do financiamento. Somente por meio de orientação clara e governação firme, os sistemas de saúde gravitam naturalmente para a finalidade de saúde para todos por meio de cuidados de saúde primários, tal como preconizava a Declaração de Alma-Ata ${ }^{(1)}$.

A efetividade da política de saúde não depende apenas das diretrizes que emanam do arcabouço jurídico-institucional, mas será garantida com a reafirmação contundente de um sistema público de saúde socialmente superior ao modelo liberal ou médico tradicional, que somente a luta constante dos movimentos sociais pelo direito à saúde pode garantir.

Agradecimentos: À Fundação de Apoio ao Ensino, Ciência e Tecnologia de Mato Grosso do Sul (FUNDECT), pelo apoio financeiro.

\section{REFERÊNCIAS}

1. Starfield B. Atenção primária: equilíbrio entre necessidades de saúde, serviços e tecnologia. Brasília: UNESCO, Ministério da Saúde; 2002.

2. Organização Panamericana da Saúde. A atenção à saúde coordenada pela APS: construindo as redes de atenção no SUS - Contribuições para o debate. Brasília: OPAS; 2011.

3. Conselho Nacional de Secretários de Saúde. Atenção Primária e Promoção da Saúde. Brasília: CONASS; 2011.

4. Elias PE. Estado e saúde: os desafios do Brasil contemporâneo.SãoPauloperspect[periódiconainternet].2004[acesso em 29 dez 2011];18(3):41-6. Disponível em: http://www. scielo.br/scielo.php?script =sci_arttext\&pid = S0102$-88392004000300005 \& \operatorname{lng}=$ pt \&nrm = isso

5. Cunha GT, Campos GWS. Apoio matricial e Atenção Primária em Saúde. Saúde Soc 2011;20(4):961-70.

6. Travassos C, Martins M. Uma revisão sobre os conceitos de acesso e utilização de serviços de saúde. Cad Saúde Pública 2004;20(Suppl 2):190-98.

7. Cunha EM, Giovanella LL. Longitudinalidade/continuidade do cuidado: identificando dimensões e variáveis para a avaliação da Atenção Primária no contexto do sistema público de saúde brasileiro. Ciênc Saúde Coletiva [periódico na internet]. 2011[acesso em 6 jan 2012]. Disponível em: http://www.scielo.br/scielo.php?script =sci arttext\&pid = S1413-81232011000700036\&lng = en

8. Baratieri T, Marcon SS. Longitudinalidade do cuidado: compreensão dos enfermeiros que atuam na estratégia saúde da família. Esc Anna Nery Rev Enferm [periódico na internet]. 2011 Dez [acesso em 6 jan 2012];15(4):802-10. Disponível em: http://www. scielo.br/scielo.php?script $=$ sci_arttext\&pid $=$ S1414$-1452011000400020 \& \operatorname{lng}=p t$

9. 9.Gomes MCPA, Pinheiro R. Acolhimento e vínculo: práticas de integralidade na gestão do cuidado em saúde em grandes centros urbanos. Interface (Botucatu) [periódico na internet]. 2005 Ago [acesso em 29 ago 2011];9(17):287-301. Disponível em: http://www. scielo.br/scielo.php? script $=$ sci arttext\&pid $=$ S1414 $-32832005000200006 \& \operatorname{lng}=\mathrm{pt}$

10. Takemoto MLS, Silva EM. Acolhimento e transformações no processo de trabalho de enfermagem em unidades básicas de saúde de Campinas, São Paulo, Brasil. Cad. Saúde Pública [periódico na internet]. 2007 Fev [acesso em 
11 jan 2012];23(2):331-40. Disponível em: http://www. scielo.br/scielo.php?script $=$ sci_arttext \&pid $=$ S0102$-311 \times 2007000200009 \& \operatorname{lng}=p t$

11. Giovanella LL, Costa LV, Carvalho AI, Conill EM. Sistemas municipais de saúde e a diretriz da integralidade da atenção: critérios para avaliação. Saúde Debate 2002;26(60):37-61.

12. Ministério da Saúde. Secretaria Geral/SESUS. Modelos assistenciais no Sistema Único de Saúde. Brasília, DF: Ministério da Saúde; 1990.

13. Mendes EV. As redes de atenção à saúde. 2. ed. Brasília: Organização Pan-Americana da Saúde; 2011.

14. Ministério da Saúde (Brasil). Portaria GM/MS n. ${ }^{\circ} 4.279$, de 30 de Dezembro de 2010. Estabelece diretrizes para a organização da Rede de Atenção à Saúde no âmbito do Sistema Único de Saúde (SUS). Diário Oficial União 30 dez 2010.

15. Ministério da Saúde (Brasil). Secretaria de Vigilância em Saúde. Departamento de Análise de Situação em Saúde. Coordenação Geral de Doenças e Agravos Não Transmissíveis. Política Nacional de Promoção da Saúde. Brasília, DF: Ministério da Saúde; 2006 [acesso em 24 dez 2011]. Disponível em: http://portal.saude.gov.br/portal/arquivos/ pdf/Politica_nacional_\%20saude_nv.pdf

16. Ministério da Saúde (Brasil). Portaria GM/MS n. ${ }^{\circ}$ 1.559, de $1^{\circ}$ de agosto de 2008. Institui a Política Nacional de Regulação do Sistema Único de Saúde. Diário Oficial União 01 ago 2008.

17. Giovanella L, Mendonça MHM, Almeida PF, Escorel S, Senna MCM, Fausto MCR, et al. Saúde da família: limites e possibilidades para uma abordagem integral de atenção primária à saúde no Brasil. Ciênc Saúde Coletiva 2009;14(3):783-94

18. Gérvas J, Fernández-Pérez M. Uma atenção primária forte no Brasil. Relatório sobre como fortalecer os acertos e corrigir as fragilidades da estratégia de saúde da família. [s.d.] [acesso em 11 jan 2012]. Disponível em: www. sbmfc.org.br/media/file/documentos/relatoriofinal_portugues.pd

19. Ministério da Saúde. Portaria GM/MS n. ${ }^{\circ}$ 1.654, de 19 de julho de 2011. Institui no âmbito do Sistema Único de Saúde, o Programa Nacional de Melhoria do Acesso e da Qualidade da Atenção Básica (PMAQ-AB) e o Incentivo Financeiro do PMAQ-AB, denominado Componente de Qualidade do Piso de atenção Básica Variável- PAB variável. Diário Oficial União 19 jul 2011.

20. Mendes EV. A APS no Brasil. In: Mendes EV, organizador. O cuidado das condições crônicas na atenção primária à saúde: o imperativo da consolidação da estratégia da saúde da família. Brasília: Organização Pan-Americana da Saúde; 2012. p. 71-99. 